\title{
A Dynamic Programming Approach for Thermal Comfort Control in Electric Vehicles
}

\author{
A. Lahlou ${ }^{1,2,3}$, F. Ossart ${ }^{1}$, E. Boudard ${ }^{2}$, F. Roy ${ }^{2}$, M. Bakhouya ${ }^{3}$ \\ ${ }^{1}$ GeePs - CNRS - CentraleSupélec - UPSud - Sorbonne Université; 91192 Gif sur Yvette - France \\ ${ }^{2}$ PSA Groupe Route de Gisy; 78943 Vélizy Villacoublay France \\ ${ }^{3}$ Université Internationale de Rabat, Faculty of Computing and Logistics, TICLab - 11100 Sala El-Jadida Maroc
}

\begin{abstract}
The HVAC system represents the main auxiliary load in electric vehicles (EVs) and requires efficient control approaches that balance energy saving and thermal comfort. In fact, passengers always demand more comfort, but on the other hand the HVAC system consumption strongly impacts the vehicle driving range, which constitutes the major concern in EVs. In this paper, dynamic programing is applied to develop an HVAC system supervisor that optimizes the thermal comfort on a given journey, for given climatic conditions and energy available. The electric vehicle model and the optimization approach are presented. Two test-cases, corresponding to hot climate, are simulated. In the first one, the energetic cost of improved comfort is quantified, while in the second one the trade-off between driving speed and thermal comfort is analyzed.
\end{abstract}

Keywords-Electric Vehicle; Thermal comfort; HVAC; energy management; Dynamic Programming.

\section{INTRODUCTION}

Energy saving and thermal comfort are two important topics in heating, ventilating and air conditioning (HVAC) control field. The HVAC system of an electric vehicle must maintain an acceptable thermal comfort inside the cabin regardless of the surrounding context. In fact, its electric consumption depends on the climatic conditions and the passengers comfort requirements, and it may be quite significant. In general, it represents $20 \%$ of the total vehicle consumption, and up to $60 \%$ in urban area and harsh conditions. Effective control approaches are therefore required to provide an acceptable balance between energy consumption and passengers' comfort [1], so as to preserve the vehicle autonomy range in given conditions.

The present paper addresses the issue of thermal comfort management and optimization for a given trip, with given climatic conditions. Dynamic programming is used to develop an off-line HVAC supervisor that optimizes the passengers' global comfort, while ensuring the EV-trip completion. The trade-off between travel time and thermal comfort is also considered, which may help the driver to adjust the vehicle driving profile accordingly.

The remainder of the paper is structured as follows. Section 2 introduces some research works related to efficient energy management systems for EVs. The HVAC system and powertrain dynamic models of EVs are presented in Section 3. In Section 4, the optimization problem is formulated, and the principle of dynamic programming is introduced. Test-case scenarios and results are presented in Section 5. Conclusions and future work are given in Section 6.

\section{RELATED WORK}

In the past few years, several approaches have been developed for managing comfort and energy consumption for either buildings or vehicles and can be categorized into two main classes. The first one aims to drive the system, at every time, to specific set points using control algorithms, while the second one has a 'supervision' role, in which the system follows trajectories that optimize comfort and energy consumption in wide time span.

\section{A. Control approaches}

A variety of control approaches for HVAC systems have been proposed and reported in the literature. Classical control has been widely adopted in building energy systems due to its simplicity in design and low computational complexity when determining the control signals. Rule-based control includes for instance on/off or bang-bang controllers, very common in old building systems without digital control. Proportional-integralderivative control is usually implemented in modern buildings, where heating and cooling systems are equipped with digital control and variable frequency drives in order to control, in the most cases, the temperature and humidity ratio [2]. The use of PID control-based approaches in automotive HVAC systems is rare. However, advanced methods from classical control theory, such as sequential quadratic programming, can handle the complexity of automotive HVAC systems [3].

One problem of PID or self-tuning PID method is that, more than one controller is required to obtain the objectives of multivarible-coupled MIMO A/C systems, which increases the design complexity of controllers. Decoupling procedure like back-stepping controllers [4] and decentralized nonlinear adaptive controllers [5] are required.

The learning-based techniques from artificial intelligence (AI) area offer a different approach to the energy management problem compared to conventional methods. AI-based control can indeed handle complex systems, deal with noisy or incomplete data, and with nonlinearities in the system. The most common AI approach is artificial neural networks (ANNs), that have been used extensively for predictions and building HVAC control strategies [6,7]. In automotive domain, an adaptive neural network tuned PID controller was proposed in [8] to improve the energy efficiency associated with the air 
conditioning unit operation. Results showed an energy reduction by almost $14 \%$.

Fuzzy logic controllers also offer a potential solution, coupling and integrating the management of all the different criteria and components of an HVAC system. Authors in [9], proposed an adaptive fuzzy output feedback controller, based on an observer for the HVAC system. A fuzzy controller for HVAC system of an automobile cabin is developed in [10]. This controller uses thermal comfort instead of temperature as its feedback. In [11] authors developed a fuzzy controller enhanced with a look ahead system that grants the air conditioning system a load-prediction ability, and a reduction of energy consumption in $\mathrm{EV}$ by $3 \%$.

Genetic algorithms (GAs) are optimization tools that can be used to improve the parameters of control techniques. In building domain, for instance, they have been extensively used for tuning the parameters of classical controllers [12] and fuzzy logic controllers [13]. Genetic algorithms can also be the solving algorithm for an optimization problem aiming to maximize comfort and safety in EVs [14].

Model predictive control has shown great potential to control HVAC systems in building and automotive domains. It is generally accepted that the reasons for this are their ability to control multivariable systems under various constraints in an optimal way. The multi-objective control, such as multivariable control (e.g., air temperature and relative humidity) [15], the decrease of operation cost (e.g. energy saving) [16] together with improvement of air quality $[17,18]$, and enhancement of steadystate performance and robustness [15,19], could be easily realized with the MPC structure.

\section{B. Supervision approaches}

In this category of approaches, dynamic programming (DP) constitute one of the most efficient algorithm to find optimal trajectories to a specific optimization problem, namely in our case, comfort and consumption optimization. These trajectories, are considered as reference trajectories, and constitute a base to develop real time controls.

Authors, in [20], proposed a dynamic programming optimization of heating load shifting, in which the ambient temperatures, solar gains, and internal loads, are known in advance. The load shifting of the customers was performed in two steps. The first step is based on the utility rate and the second step depends on the amount of carbon emission. Load shifting was performed within a small indoor temperature variation to maintain the customer comfort and save energy cost from heating the building within $19{ }^{\circ} \mathrm{C}$. Obtained results show the effectiveness of DP, as a supervision algorithm, in maintaining a good balance between energy consumption and comfort, and constitute a first step to develop controls that will follow state trajectories computed by DP.

In this work, we investigate DP to develop an energy management system based on an optimal compromise between thermal comfort and energy consumption in electric vehicle. Before introducing the proposed approach, we present the model of different parts of the system (HVAC system, powertrain and battery), and specify the comfort criteria.

\section{EV MODELING}

This section presents the system model, which is composed of three sub-systems: the HVAC system, the powertrain and the battery, which feeds both of them.

\section{A. HVAC system model}

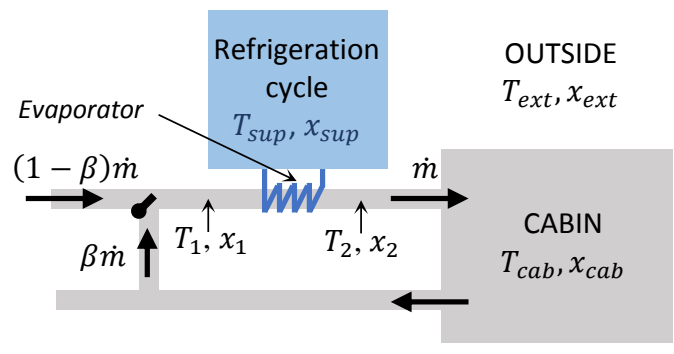

Fig. 1 Schematic diagram of the HVAC system

The HVAC system diagram is shown in Fig. 1. The outside air and recycled cabin air flows are mixed, blown and cooled through the refrigeration cycle evaporator, before being injected into the cabin zone. The air flow rate is denoted by $\dot{m}$ and $\beta$ represents the fraction of recycled cabin air in this flow. Heat exchanges between the cabin and the external environment take place by convection, air circulation and solar irradiation. $T$ represents the temperature while $x$ refers the specific humidity, which is defined as the mass of water vapor present in a unit mass of moist air. These quantities are defined at different points of the system: before and after the evaporator, at the condenser surface (subscript sup), in the cabin, and outside the cabin.

The cabin temperature evolution is governed by (1), where $C_{c a b}$ is the cabin thermal capacitance (including walls and seats). $P_{\text {conv }}$ is the thermal power exchanged through the windshield and walls by convection. It is given by (2), where $H$ is the convection factor. $P_{\text {enth }}$ corresponds to the heat exchange due to air circulation and given by (3), where $h_{2}$ (resp. $h_{\text {hab }}$ ) denotes the massic enthalpy of the air blown into the cabin (resp. extracted from the cabin), which is calculated according to (4). $P_{\text {sol }}$ represents the solar irradiation power and solely depends on meteorological conditions.

$$
\begin{gathered}
C_{c a b} \frac{d T_{c a b}}{d t}(t)=P_{\text {conv }}(t)+P_{\text {enth }}(t)+P_{\text {sol }}(t) \\
P_{\text {conv }}(t)=H \cdot\left[T_{\text {ext }}(t)-T_{h a b}(t)\right] \\
P_{\text {enth }}(t)=\dot{m} \cdot\left[h_{2}(t)-h_{h a b}(t)\right] \\
h(T, x)=1006 . T+x \cdot(2500130+1823 . T)
\end{gathered}
$$

The cabin specific humidity evolves according to the water mass balance equation (5), where $V_{c a b}$ and $\rho_{\text {air }}$ denote the cabin volum and the air density respectively.

$$
V_{c a b} \cdot \rho_{a i r} \cdot \frac{d x_{c a b}}{d t}=\dot{m} \cdot\left[x_{2}-x_{c a b}\right]
$$

Part of the return air from the cabin is mixed with the outside air and re-circulated back. The temperature and the specific humidity of the mixed air are given by (6) and (7), where the 
recycling ratio $\beta$ is controlled according to the external temperature.

$$
\begin{aligned}
& T_{1}=\beta T_{c a b}+(1-\beta) T_{\text {ext }} \\
& x_{1}=\beta x_{c a b}+(1-\beta) x_{\text {ext }}
\end{aligned}
$$

The mixed air is blown through the evaporator of the refrigeration cycle, where it is cooled and dried. The evaporator is one of the four components of the refrigeration cycle, the others being the compressor, the condenser and the thermal expansion valve [21]. In the evaporator, the refrigerant liquid absorbs heat from the air, producing a temperature drop and condensation. The temperature and specific humidity after the evaporator are given by equations (8) and (9), where $\eta$ is the evaporator efficiency, $T_{\text {sup }}$ and $x_{\text {sup }}$ are the evaporator superficial temperature and specific humidity. $T_{\text {sup }}$ is approximately equal to the refrigerant temperature, which is controlled by the compressor speed.

$$
\begin{aligned}
& T_{2}=T_{1}-\eta \cdot\left(T_{1}-T_{\text {sup }}\right) \\
& x_{2}=x_{1}-\eta \cdot\left(x_{1}-x_{\text {sup }}\right)
\end{aligned}
$$

The cooling power absorbed by the refrigerant liquid is given by (10), where $C_{p}$ is the air specific heat capacity and $L$ is the water latent heat of condensation.

$$
P_{\text {cool }}=\dot{m}\left[C_{p}\left(T_{2}-T_{1}\right)+L\left(x_{2}-x_{1}\right)\right]
$$

The refrigerant cycle needs energy in order to compress and pump the refrigerant liquid. The electric consumptions of the different components depend on the external temperature according to tabulated data provided by PSA Group. The total electric consumption of the HVAC system can be formulated in the following synthetic form $P_{H V A C}\left(P_{c o o l}, T_{\text {ext }}\right)$.

Despite its simplicity, the previous model is accurate enough for our purpose: it allows to calculate the influence of the system control variable $T_{\text {sup }}$ on the cabin temperature and humidity, and the corresponding HVAC electrical power consumption.

\section{B. Powertrain model}

The second sub-system is the powertrain. The electrical machine provides a torque $T_{E M}$, which is transmitted to the wheels and converted there into a tractive force $F_{\text {traction. }}$. The resulting vehicle speed evolution is calculated according to (11), where $m$ is the vehicle equivalent mass, accounting for all moving parts, and $F_{\text {road }}$ denotes the sum of the external forces the vehicle is subjected to: aerodynamic drag, gravitational force, and rolling $v$ [22,23]. Ftraction is negative during regenerative braking.

$$
m \cdot \frac{d v}{d t}(t)=F_{\text {traction }}(t)+F_{\text {road }}(t)
$$

The transmission chain between the electrical machine and the wheels is modelled by a fixed speed ratio and a variable efficiency given by tabulated data as a function of torque and speed. The electrical machine and its control electronics are modelled by a measured losses map. Hence, the electrical machine power consumption $P_{E M}$ is given by (12), where $\omega_{E M}$ denotes the machine rotation speed, which is proportional to the vehicle speed.

$$
P_{E M}=T_{E M} \omega_{E M}-\operatorname{losses}\left(T_{E M}, \omega_{E M}\right)
$$

In the present study, we consider that the driver is modeled as a PI regulator which controls the electrical machine torque in order to follow the speed profile of a given driving cycle. The actual speed profile $v(t)$ and the resulting electrical consumption $P_{E M}(t)$ are calculated according to the equations afore mentioned.

\section{Battery model}

The battery provides energy to the traction machine, the HVAC system and various auxiliaries, according to (13).

$$
P_{b a t}=P_{E M}+P_{H V A C}+P_{a u x}
$$

The battery is modeled by its open circuit voltage $V_{b a t}$ and internal resistance $R_{\text {bat }}$. Formula (14) and (15) give the current as a function of the power and the resulting battery state of energy $S O E$ variation. $E_{0}$ is the nominal battery energy.

$$
\begin{gathered}
i_{b a t}(t)=\frac{V_{b a t}-\sqrt{V_{b a t}{ }^{2}-4 R_{b a t} P_{b a t}(t)}}{2 R_{b a t}} \\
\frac{d S O E}{d t}(t)=\frac{V_{b a t}}{E_{0}} i_{\text {bat }}(t)
\end{gathered}
$$

\section{OPTIMAL ENERGY MANAGEMENT}

The objective of the HVAC management system is to control the cabin temperature and relative humidity, so that the passengers feel comfortable at the lowest energy cost. On the other hand, the driver first priority is to reach his destination, and the battery state of charge may be too low to insure ideal comfort during the whole trip. Hence, it may be necessary to limit the power provided to air conditioning, in order to extend the vehicle driving range up to its final destination. This energy management problem can be described as an optimal control problem and we propose to use dynamic programing to solve it. In the rest of this section, we first introduce the comfort criteria and then formulate the optimal energy management problem.

\section{A. Thermal comfort criteria}

Thermal comfort is a complex notion that involves the surrounding temperature and the relative humidity as main factors. At a given pressure, the relative humidity, denoted by $\mathrm{HR}$, depends on the temperature and the specific humidity $x$ calculated by our model. As a first approximation, thermal comfort is defined by temperature and relative humidity ranges perceived as being comfortable by the vehicle passengers, as expressed by (16) and (17).

$$
\begin{gathered}
T_{\text {ref }}-0.5 \Delta \mathrm{T} \leq \mathrm{T} \leq T_{\text {ref }}+0.5 \Delta \mathrm{T} \\
H R_{\text {ref }}-0.5 \Delta \mathrm{HR} \leq \mathrm{HR} \leq H R_{\text {ref }}+0.5 \Delta \mathrm{HR}
\end{gathered}
$$

Typical values used by PSA Group are $: T_{r e f}=25^{\circ} \mathrm{C}$ with $\Delta \mathrm{T}=$ $1^{\circ} \mathrm{C}$ and $H R_{\text {ref }}=45 \%$ with $\Delta \mathrm{HR}=30 \%$. As a first approximation, we propose a quadratic discomfort criterion defined by (18).

$$
L(T, H R)=0.5\left(\frac{\mathrm{T}-T_{r e f}}{\Delta \mathrm{T}}\right)^{2}+0.5\left(\frac{\mathrm{HR}-H R_{r e f}}{\Delta H R_{r e f}}\right)^{2}
$$




\section{B. Problem formulation}

The role of the proposed energy management is to determine the best HVAC system control, in order to reach the lowest thermal discomfort for a given trip. The trip is modeled by a driving cycle, known in advance. The powertrain model is used to calculate the instantaneous power that will be required by the electric machine $P_{E M}(t)$, and then the total energy required for traction over the considered trip. The difference between the energy embedded in the battery at the beginning of the trip and the energy needed for traction gives the energy available for air conditioning.

The control and the state variable are defined by $u=\left[T_{\text {sup }}\right]^{\prime}$ and $x=\left[T_{c a b} H R_{c a b} S O E\right]^{\prime}$ respectively. The outside weather, assumed to be known, is included in the disturbance variable defined by $w=\left[T_{\text {ext }} R H_{\text {ext }} P_{\text {sol }} P_{E M}\right]^{\prime}$. With these notations, the system's dynamic equation described in sections III.A and III.C can be rewritten according to (19).

$$
\dot{x}(t)=f(x(t), u(t), w(t))
$$

An important operational constraint is the battery power limitation, expressed as an interval constraint (20). When $P_{E M}$ reaches the battery limit, there is no power left for air conditioning. Other operational constraints are active, but they are not all detailed here.

$$
\left|P_{\text {bat }}(t)\right| \leq P_{\text {bat max }}
$$

The optimization problem consists in determining the command that minimizes the global discomfort (21) on the considered trip.

$$
J(u)=\int_{0}^{t_{f i n}} L\left(T_{c a b}(t), H R_{c a b}(t)\right) d t
$$

The finite energy constraint is an inequality end constraint (22).

$$
\operatorname{SOE}\left(t_{\text {fin }}\right) \geq 0
$$

This optimization problem can be numerically solved using a dynamic programming algorithm. Dynamic programming is a sequential optimization method, which provides an optimal set of commands over the considered time interval. It consists in a recursive optimization procedure, which builds the solution of the overall $\mathrm{N}$-stage problem by first solving a one-stage problem and sequentially including one stage at a time until the overall optimum is found. Usually, computations are done backward, starting from the final state. In our case, the final state is not known, which enforce us to do a forward dynamic programming.

\section{Simulation Results}

This section focuses on the evaluation of the proposed approach and shows its effectiveness in minimizing and managing thermal discomfort on a given trip, for given meteorogical conditions. Two scenarios are considered. The first one highlights the tradeoff that can be achieved between energy consumption and thermal comfort. The second one illustrates how the speed profile can be adjusted to improve thermal comfort.

\section{A. Simulation data and technical characteristic of the vehicle}

For both scenarios, we considered an electric vehicle initially parked outside and used to make a $115 \mathrm{~km}$ trip lasting about one hour. The driving cycle is the succession of four highway Artemis driving cycles. The weather conditions are very hot, with an outside temperature $T_{\text {ext }}$ between $33^{\circ} \mathrm{C}$ and $34^{\circ} \mathrm{C}$, as shown in Fig. 3, and a constant $50 \%$ relative humidity rate. The solar radiation is correlated with the outside temperature, equal to $900 \mathrm{~W} / \mathrm{m}^{2}$ when $T_{\text {ext }}=34{ }^{\circ} \mathrm{C}$ and $1000 \mathrm{~W} / \mathrm{m}^{2}$ for $T_{\text {ext }}=35^{\circ} \mathrm{C}$. The initial temperature and relative humidity in the cabin are respectively $37^{\circ} \mathrm{C}$ and $50 \%$.

The vehicle corresponds to a small size car, with a nominal power around $60 \mathrm{~kW}$ and a $40 \mathrm{kWh} \mathrm{Li-ion} \mathrm{battery.} \mathrm{Fig.} 2$ shows that at high speed, due to power limitations, the actual vehicle speed (red curve) does not exactly follow the Artemis cycle. At these times, the HVAC system must be turned off.

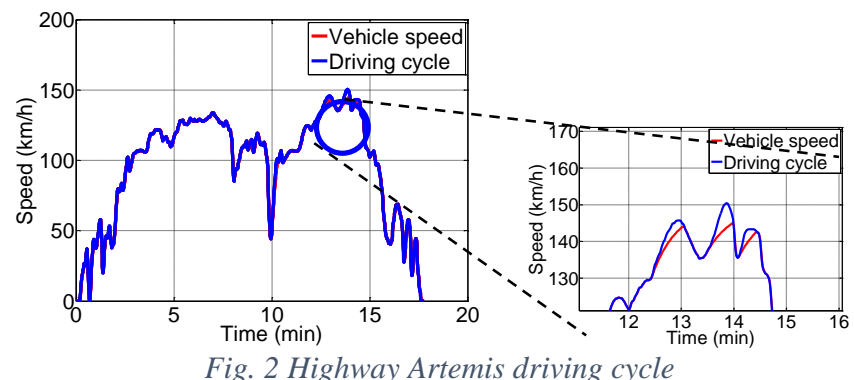

\section{B. Scenario 1: Thermal discomfort vs energy cost trade-off}

In this scenario, we consider different initial battery state of charge. The battery energy is sufficient to ensure the traction on the entire trip ( $23 \mathrm{kWh}=59 \%)$, but there is more or less energy available for air conditioning. The DP algorithm computes the control that optimizes the thermal comfort according to the energy available for air conditioning.

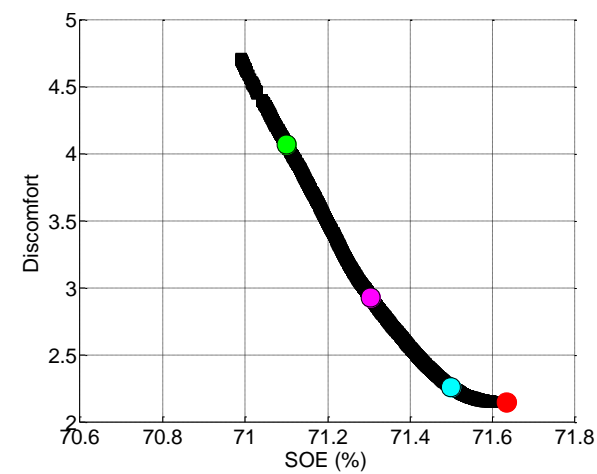

Fig. 3 Pareto frontier: thermal discomfort versus initial battery state of energy (SOE). The temperature and the humidity profiles corresponding to the colored dots are plotted in Fig. 4

Fig. 3 shows the resulting thermal discomfort as a function of the initial battery state of energy. This curve corresponds to the Pareto frontier, with respect to the thermal comfort and energy consumption criteria. For an initial SOE below $70.6 \%$, there is not enough energy to keep the HVAC system on until the end of the trip. For an initial SOE between $71.1 \%$ and $71.63 \%$, the 
thermal discomfort decreases, as there is more energy available. For example, the discomfort is reduced from 4 to 2.2 at an additional energy cost of $200 \mathrm{Wh}(0.5 \%$ of the battery capacity).
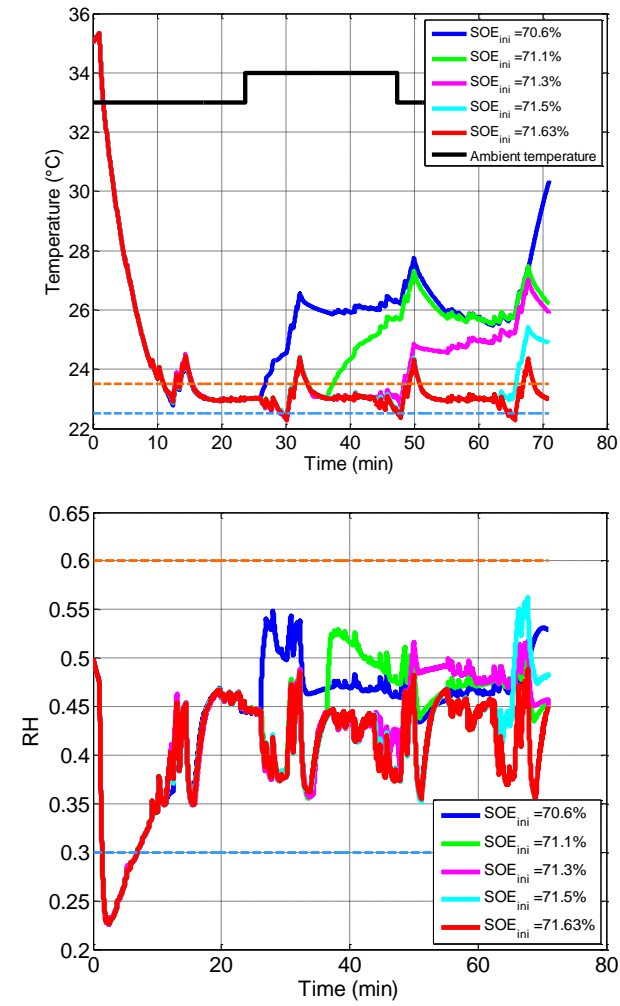

Fig. 4 Cabin temperature and relative humidity trajectories computed by DP, for different initial battery SOE.

Fig. 4 shows the evolution of the temperature and the relative humidity in the passenger compartment during the trip, for different initial state of energy (colored marks of Fig. 3). As already underlined, each trajectory corresponds to the best reachable comfort for the considered energy. The most uncomfortable and energy-consuming phase takes place at the beginning of the trip, when the hot cabin needs to be cooled down. This phase cannot be reduced below a certain time due to the thermal inertia of the system and the maximum power of the air conditioner. The temperature is afterwards maintained at best around the temperature set point. We note that the temperature rises when the HVAC is turned off due to battery power limitations, and that the dynamic programming algorithm anticipates this temporary lack of power by lowering the cabin temperature in order to maintain at best the temperature in the comfort zone.

\section{Scenario 2: Travel time vs thermal comfort trade-off}

In the second scenario, the driving cycle is modified, so that the driver travels the same distance at a lower speed. Less energy is required for traction, and more is available for air conditioning.

A scaling factor $k<1$ is applied to the speed as follows:

$$
v_{\text {new }, \text { cycle }}(t)=v_{\text {cycle }}(t) \cdot k
$$

Simulations have been conducted for a given initial SOE equal to $71.1 \%$, which does not allow to reach optimal comfort over the considered trip. By decreasing the values of $k$, one reduces the power needed for traction and comfort is improved, as shown by Fig. 5 . The cabin temperature and relative humidity are closer to ideal values all over the journey. If the driver travels at a lower speed, accepting to increase the trip duration, he can improve the thermal comfort because there is more energy available for air conditioning. By driving at lower speed, he also avoids reaching the battery power limit, and hence undesired HVAC switching off. This can be seen in the reduced amplitude of temperature rises throughout the cases.
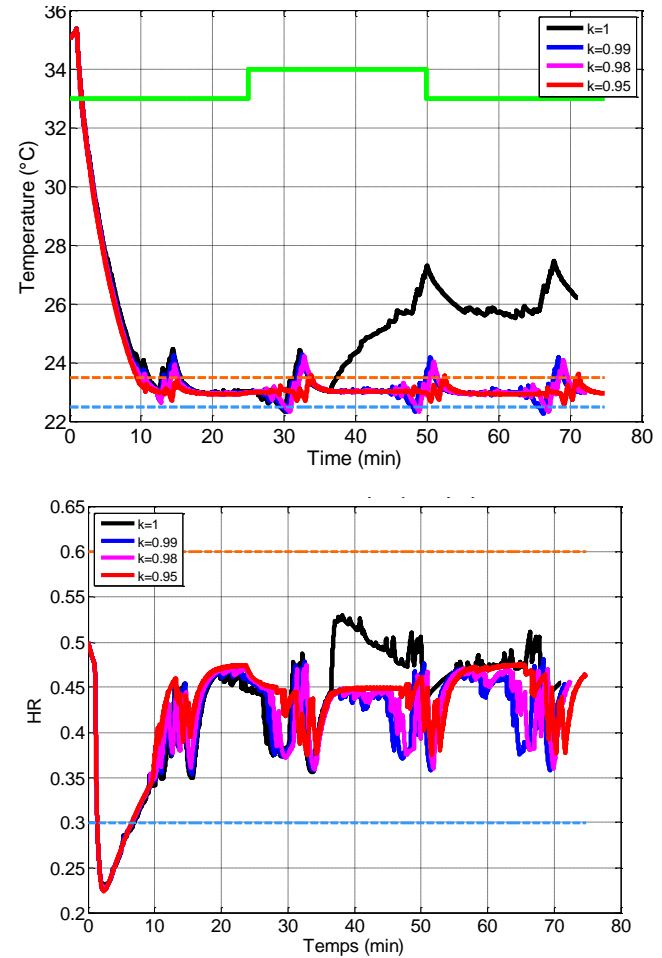

Fig. 5 Cabin temperature and relative humidity trajectories computed by DP, for different values of the parameter $k$

Table I summarizes the results: the traction, the AC and the surplus energies, together with the thermal discomfort as a function of the time travel increase. At first, the whole battery energy is needed to improve the comfort, but when slowing down, optimal comfort is reached without using the whole battery energy. Fig. 6 gives a graphical representation of these results.

TABLE I. ENERGIES (KW.H) AND THERMAL DISCOMFORT

\begin{tabular}{ccccc}
$\Delta t(\%)$ & $\begin{array}{c}\text { Traction } \\
\text { energy } \\
(\boldsymbol{k W h})\end{array}$ & $\begin{array}{c}\text { AC } \\
\text { energy } \\
(\boldsymbol{k W h})\end{array}$ & $\begin{array}{c}\text { Surplus } \\
\text { energy } \\
(\boldsymbol{k W h})\end{array}$ & $\begin{array}{c}\text { Discomfort } \\
(\boldsymbol{a} . \boldsymbol{u})\end{array}$ \\
\hline 0 & 23.01 & 2.69 & 0 & 4.06 \\
0.60 & 22.84 & 2.85 & 0 & 2.26 \\
0.81 & 22.79 & 2.9 & 0 & 2.09 \\
1.01 & 22.74 & 2.94 & 0.02 & 2.03 \\
2.04 & 22.45 & 2.99 & 0.26 & 1.98 \\
5.26 & 21.56 & 3.13 & 1.01 & 1.85
\end{tabular}




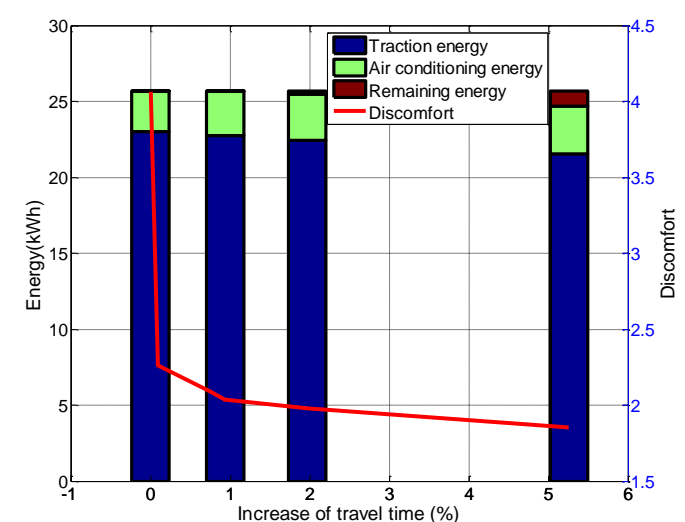

Fig. 6 Energy and discomfort versus travel time increase

\section{CONCLUSION AND FUTUR WORK}

This paper presented an optimal control of comfort in EV cabin by using a dynamic programming approach. Simulations have been conducted in two different scenarios and results show that the cabin comfort can be improved if a small additional amount of energy is allocated for air conditioning, in driving contexts with hot weather.

The results show also the usefulness of using PD for thermal comfort management. Despite that the thermal comfort criterion used is too simple to be qualitatively interpreted our ongoing work will focus on evaluating thermal comfort by indexes, which account for thermo-physiologic phenomena in human body.

\section{ACKNOWLEDGMENTS}

This work is supported by PSA Groupe, the French ANRT and the Morrocan CNRST, under the joint France-Morocco CIFRE program, and is performed under the collaborative framework OpenLab "PSA@Paris-Saclay - Electrical engineering for Mobility" and OpenLab "PSA@Morocco - Sustainable mobility for Africa".

\section{REFERENCES}

[1] Valentina, R.; Viehl, A.; Bringmann, O.; Rosenstiel, W. HVAC system modelling for range prediction of Electric Vehicles. In Proceedings of the Intelligent Vehicles Symposium, Dearborn, MI, USA, 8-11 June 2014; pp. $1145-1150$.

[2] C. Blasco, J. Monreal, I. Benitez, A. Lluna, Modelling and PID control of HVACsystem according to energy efficiency and comfort criteria, Sustainability inEnergy and Buildings. Smart Innovation, Systems and Technologies, vol. 12,2012.

[3] Chiu C-C, Tsai N-C, Lin C-C. Near-optimal order-reduced control for $\mathrm{A} / \mathrm{C}$ (air-conditioning) system of EVs (electric vehicles). Energy 2014;66:342-53.

[4] Semsar, E.; Yazdanpanah, M.J.; Lucas, C. Nonlinear Control and Disturbance Decoupling of an HVAC System via Feedback Linearization and Back-Stepping. In Proceedings of the 2003 IEEE Conference on Control Applications (CCA 2003), Istanbul, Turkey, 23-25 June 2003; pp. 646-650.
[5] Huaguang, Z.; Cai, L. Decentralized nonlinear adaptive control of an HVAC system. IEEE Trans. Syst. Man Cybern. Part C Appl. Rev. 2002, 32, 493-498.

[6] Beghi, A.; Cecchinato, L.; Rampazzo, M.; Simmini, F. Load forecasting for the efficient energy management of HVAC systems. In Proceedings of the 2010 IEEE International Conference on Sustainable Energy Technologies (ICSET), Kandy, Sri Lanka, 6-9 December 2010; pp. 1-6.

[7] Curtiss, P.S. Examples of Neural Networks Used for Building System Control and Energy Management. In Proceedings of the American Society of Heating, Refrigerating and Air-Conditioning Engineers (ASHRAE) Annual Meeting, Boston, MA, USA, 28 June-2 July 1997; Volume 103, pp. 909-913.

[8] Khayyam H, Kouzani A Z, Hu E J, et al. Coordinated energy management of vehicle air conditioning system[J]. Applied Thermal Engineering, 2011, 31, 5: 750-764.M. Young, The Technical Writer's Handbook. Mill Valley, CA: University Science, 1989.

[9] Baek, J.; Kim, E.; Park, M. Adaptive Fuzzy Output Feedback Control for the Nonlinear Heating, Ventilating, and Air Conditioning System. In Proceedings of the 23rd International Technical Conference on Circuits/Systems, Computers and Communications (ITC-CSCC 2008), Shimonoseki, Japan, 6-9 July 2008; pp. 1477-1480.

[10] Farzaneh, Y., Tootoonchi, A. A. (2008) Intelligent Control of Thermal Comfort in Automobile, 2008 IEEE Cybernetics and Intelligent Systems, 21-24 Sept.

[11] Khayyam H, Nahavandi S, Hu E, et al. Intelligent energy management control of vehicle air conditioning via look-ahead system[J]. Applied Thermal Engineering, 2011, 31, 16: 3147-3160.

[12] Huang, W.; Lam, H.N. Using genetic algorithms to optimize controller parameters for HVAC systems. Energy Build.1997, 26, 277-282.

[13] Alcala, R.; Benitez, J.M.; Casillas, J.; Cordon, O.; Perez, R. Fuzzy control of HVAC systems optimized by genetic algorithms. Appl. Intell. 2003, 18, 155-177.

[14] M. Busl, T. Sattelmayer, and W. Polifke, "Design of an Energy-Efficient Climate Control Algorithm for Electric Cars," Department of Automatic Control, Lund University, Sweden, 2011

[15] ] Oldewurtel F, Parisio A, Jones CN, Gyalistras D, Gwerder M, Stauch V, et al. Use of model predictive control and weather forecasts for energy efficient building climate control. Energy Build 2012;45:15e27.

[16] H. Yonghong, L. Nianping, and Y. Yixun, "Fuzzy model predictive control for a comfort air-conditioning system," Proceeding of the 2006 IEEE International Conference on Automation Science and Engineering, Shanghai China, pp. 530-533, October 2006,

[17] M. Razi, M. Farrokhi, M. H. Saeidi, and et al, "Neuro-predictive control for automotive air conditioning system," ICEIS Proc. Sixth Int. Conf. Enterp. Inf. Syst, Islamabad Pakistan, pp. 1-6, April 2006.

[18] Mei, J., \& Xia, X. (2017). Energy-efficient predictive control of indoor thermal comfort and air quality in a direct expansion air conditioning system. Applied Energy, 195, 439-452. http://dx.doi.org/10.1016/j.apenergy.2017.03.076.

[19] H. Gongsheng, W Shengwei, and X. Xinhua, "A robust model predictive control strategy for improving the control performance of air-conditioning systems," Energy Conversion and Management, vol. 50, pp. 2650-2658, 2009.

[20] Favre B, Peuportier B. Application of dynamic programming to study load shifting in buildings. Energy Build 2014;82:57-64.

[21] Howell, R.H., Sauer, Jr, H.J., and Coad, W.J., 1998, Principles of Heating, Ventilating, and Air Conditioning, American Society of Heating, Refrigerating and Air-Conditioning Engineers, Atlanta.

[22] Erik Schaltz. Electrical Vehicle Design and Modeling, Electric Vehicles - Modelling and Simulations(2011), Dr. Seref Soylu (Ed.), ISBN: 978953-307-477-1, InTech, Available from:http://www.intechopen.com/books/electric-vehicles-modellingandsimulations/electrical-vehicle-design-andmodeling.

[23] F. Salem, "Modeling and control solutions for electric vehicles," European Scientific Journal,9 (15), 221-240, 2013. 